\title{
Application of Feedforward Control to A Vibration Isolation System Using Negative Stiffness Suspension*
}

\author{
Md. Emdadul HOQUE**, Takeshi MIZUNO**, Masaya TAKASAKI** \\ and Yuji ISHINO** \\ **Department of Mechanical Engineering, Saitama University \\ 255 Shimo-Okubo, Sakura-Ku, Saitama 338-8570, Japan \\ E-mail: mehoque@mech.saitama-u.ac.jp
}

\begin{abstract}
This paper presents a vibration isolation system using negative stiffness suspension. The vibration isolation system is developed by combining a positive stiffness suspension in series with a negative stiffness suspension. The developed system could realize zero-compliance to direct disturbance, as well as ground vibration isolation. In the previous system, the ground vibration and, the static and low frequency sinusoidal direct disturbances were efficiently suppressed. However, there was a reverse action in case of impulse or step wise disturbance. Therefore, a peak was appeared which was, sometimes, larger than the displacement caused by the step load. This unpleasant response might hamper the objective function of many advanced systems. In this research, a feedforward controller is added in combination with zero-power control to overcome this problem. Some experimental results are presented to verify the proposed control system in a vibration isolation apparatus.
\end{abstract}

Key words: Active Control, Vibration Isolation, Magnetic Suspension, Mechatronics

\section{Introduction}

The researches on vibration isolation and control have attracted significant attention recently due to their broad and potential applications in advanced technologies including advanced micro manufacturing and space technology. It is equally important to insulate vibrations from two kinds of sources, such as direct disturbance on the table and ground vibration. Passive technique can be employed by utilizing stiff spring or suspension for suppressing the direct disturbance and by using soft spring or suspension for ground vibration isolation. However, a trade-off between them is vital for attenuating both disturbances $^{(1),(2)}$. To overcome the above dilemma, an active vibration control has been an unquestionable choice. Furthermore, only passive technique is not sufficient to achieve high performance $^{(3)-(8)}$.

PID, LQR, $\mathrm{H}_{\infty}$ control, PI control, repetitive control, pole placement have extensively been used in the active feedback control systems. However, with the advanced technology of semiconductor devices and high-density recording, precise vibration isolation systems have increasingly been demanded. For example, the silicon wafer photolithography system, a principal equipment in the semiconductor manufacturing process, has a stage which is moved in steps and causes direct disturbance. Suppression of the transient response to such disturbance is a major problem in the process ${ }^{(3)}$. A number of isolation tables with

${ }^{*}$ Received 22 Feb., 2011 (No. 11-0137) [DOI: 10.1299/jsdd.5.777]

Copyright $@ 2011$ by JSME 
feedforward control have also been developed to reduce the response of the isolation table ${ }^{(8)-(11)}$. However, most of the active vibration isolation systems use high-performance sensors, such as servo-type accelerometers to detect the vibration signal of an isolation table. Apart from this, large amount of control current is necessary to drive the actuators. It makes the system rather expensive than the passive systems. This is one of the obstacles to expand the field of application of active vibration control.

To overcome this, vibration isolation systems have been developed using active zero-power controlled magnetic suspension ${ }^{(12),(13)}$. In the proposed system, eddy-current relative displacement sensors can be used for displacement feedback. Moreover, the control current converges to zero for the zero-power control system. Therefore, no control current is necessary to drive the actuator in the steady state. The above systems use a combined system with positive and negative springs in series. A middle table is introduced in this system between the base and the isolation table. Positive stiffness spring is used between the base and the middle table, and a suspension with negative stiffness is used between the middle table and the isolation table.

In this research, an active zero-power control is used to realize negative stiffness by using a hybrid magnet consists of electromagnet and permanent magnets. This control achieves the steady state in which the attractive force produced by the permanent magnets balance the weight of the suspended object, and the control current converges to zero. Since there is no steady energy consumption for achieving stable levitation, it has been applied to space vehicles ${ }^{(14)}$, and to the magnetically levitated carrier system in clean rooms ${ }^{(15)}$. Moreover, it can be noted that realizing negative stiffness can also be generalized by using linear actuator (voice coil motor) instead of hybrid magnet ${ }^{(16)}$.

A unique characteristic of the zero-power control is that it realizes negative stiffness ${ }^{(17)}$. Therefore, a very high (theoretically infinite) stiffness of a vibration isolation system can be achieved by combining a mechanical spring in series with a zero-power control. In the previous system, zero-compliance of the isolation table is realized when displacement of the middle table is equal to the change of gap between the middle table and the isolation table. It enables the developed system to have good characteristics against both disturbances. However, the results of step responses show that the isolation table is displaced from the original position in the transient period ${ }^{(18)}$. In this case, active feedback control may not achieve sufficient high-speed responses, because of the delays contained inherently in the control scheme. To diminish this transient response, higher closed-loop poles can be used in the zero-power controller ${ }^{(18)}$. If too much higher closed-loop poles are chosen, the controller would be fast, but unstable. Furthermore, it would deteriorate vibration isolation characteristics.

Therefore, the objective of this paper is to introduce a feedforward controller in combination with zero-power control system. It is demonstrated that the transient response of the isolation table, emerged as a large peak due to zero-power control and PD control, can be diminished by the inclusion of the feedforward control.

\section{Negative Stiffness Suspension}

Negative stiffness is generated by actively controlled zero-power magnetic suspension. The basic model, controller and the characteristic of the zero-power control system is described below.

\subsection{Model}

A basic zero-power controller is designed for simplicity based on linearized equation of motions. It is assumed that the displacement of the suspended mass is very small and the nonlinear terms are neglected. The suspended object with a mass of $m$ is assumed to move only in the vertical translational direction as shown by Fig. 1. The equation of motion is 


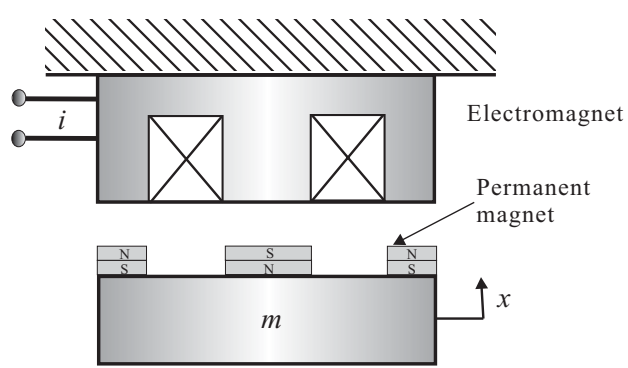

Fig. 1 A model of zero-power controlled magnetic suspension

given by

$$
m \ddot{x}=k_{s} x+k_{i} i+w,
$$

where $x$ : displacement of the suspended object from the equilibrium position, $k_{s}$ : gap-force coefficient of the permanent magnet, $k_{i}$ : current-force coefficient of the electromagnet, $i$ : control current, $w$ : disturbance acting on the suspended object. The equilibrium position of the suspended object is derived as the position where the gravitational force and the force of the electromagnet are balanced. The coefficients $k_{s}$ and $k_{i}$ are positive. When each Laplace-transform variable is denoted by its capital, and the initial values are assumed to be zero for simplicity, the transfer function representation of the dynamics described by Eq. (1) becomes

$$
X(s)=\frac{1}{m s^{2}-k_{s}}\left(k_{i} I(s)+W(s)\right)=\frac{1}{s^{2}-a_{0}}\left(b_{0} I(s)+d_{0} W(s)\right),
$$

where $a_{0}=k_{s} / m, b_{0}=k_{i} / m$, and $d_{0}=1 / m$.

\subsection{Zero-Power Controlled Magnetic Suspension}

Zero-power can be achieved either by feeding back the velocity of the suspended object or by introducing a minor feedback of the integral of current in the PD (proportional-derivative) control system ${ }^{(17)}$. Since PD control is a fundamental control law in magnetic suspension, zero-power control is realized from PD control in this research using the second approach. In the current controlled magnetic suspension system, PD control can be represented as

$$
I(s)=-\left(p_{d}+p_{v} s\right) X(s),
$$

where $p_{d}$ : proportional feedback gain, $p_{v}$ : derivative feedback gain. Figure 2 shows the block diagram of a current-controlled zero-power controller where a minor integral feedback of current is added to the PD control.

The control current of zero-power controller is given by

$$
I(s)=-\left(p_{d}+p_{v} s\right) X(s)+\frac{p_{z}}{s} I(s)
$$

where $p_{z}$ : integral feedback gain in the minor current loop. From Eqs. (2) to (4), it can be written as

$$
\begin{aligned}
& \frac{X(s)}{W(s)}=\frac{\left(s-p_{z}\right) d_{0}}{s^{3}+\left(b_{0} p_{v}-p_{z}\right) s^{2}+\left(b_{0} p_{d}-a_{0}\right) s+a_{0} p_{z}}, \\
& \frac{I(s)}{W(s)}=\frac{-s\left(s p_{v}+p_{d}\right) d_{0}}{s^{3}+\left(b_{0} p_{v}-p_{z}\right) s^{2}+\left(b_{0} p_{d}-a_{0}\right) s+a_{0} p_{z}} .
\end{aligned}
$$




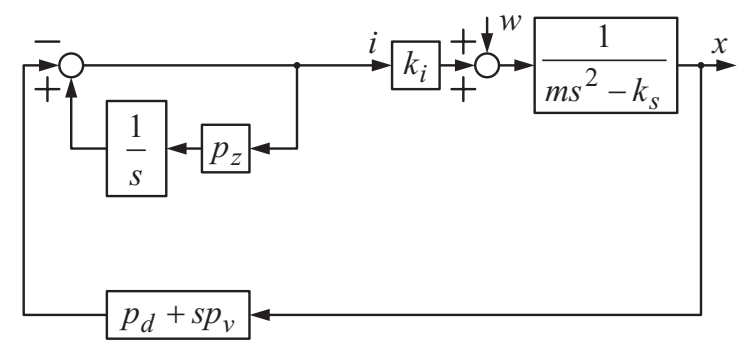

Fig. 2 Block diagram of the zero-power controller

To estimate the stiffness for direct disturbance, the direct disturbance, $W(s)$ on the isolation table is considered to be stepwise, that is

$$
W(s)=\frac{F_{0}}{s},\left(F_{0}: \text { constant }\right) .
$$

From Eqs. (6) and (7)

$$
\lim _{t \rightarrow \infty} i(t)=\lim _{s \rightarrow 0} s I(s)=0 .
$$

It indicates that control current always converges to zero in the zero-power control for any load. The steady displacement of the suspension, from Eqs. (5) and (7), is given by

$$
\lim _{t \rightarrow \infty} x(t)=\lim _{s \rightarrow 0} s X(s)=-\frac{d_{0}}{a_{0}} F_{0}=-\frac{F_{0}}{k_{s}} .
$$

The negative sign in the right-hand side illustrates that the new equilibrium position is in the direction opposite to the applied force. It means that the system realizes negative stiffness. Assume that stiffness of any suspension is denoted by $k$. The stiffness of the zero-power controlled magnetic suspension is, therefore, negative and given by

$$
k=-k_{s} .
$$

\subsection{Stiffness Adjustment}

The stiffness realized by zero-power control is constant, as shown in Eq. (10). However, it is necessary to adjust stiffness of the zero-power control system in many applications, such as vibration isolation systems. There are two approaches to adjust stiffness of the zero-power control system. The first one is by adding a minor displacement feedback gain to the zero-power control current, and the other one is by adding a proportional feedback in the minor current feedback loop ${ }^{(19)}$. In this research, stiffness adjustment capability of zero-power control is realized by the first approach. Figure 3 shows the block diagram of the modified zero-power controller that is capable to adjust stiffness. The control current of the modified zero-power controller is given by

$$
I^{\prime}(s)=-\left(\frac{p_{d} s}{s-p_{z}}+\frac{p_{v} s^{2}}{s-p_{z}}+p_{s}\right) X(s),
$$

where $p_{s}$ : proportional displacement feedback gain across the zero-power controller.

The transfer-function representation of the dynamics shown in Fig. 3 is given by

$$
\frac{X(s)}{W(s)}=\frac{\left(s-p_{z}\right) d_{0}}{s^{3}+\left(b_{0} p_{v}-p_{z}\right) s^{2}+\left(b_{0} p_{d}+b_{0} p_{s}-a_{0}\right) s+a_{0} p_{z}-b_{0} p_{s} p_{z}} .
$$

From Eqs. (12) and (7), the steady displacement becomes 


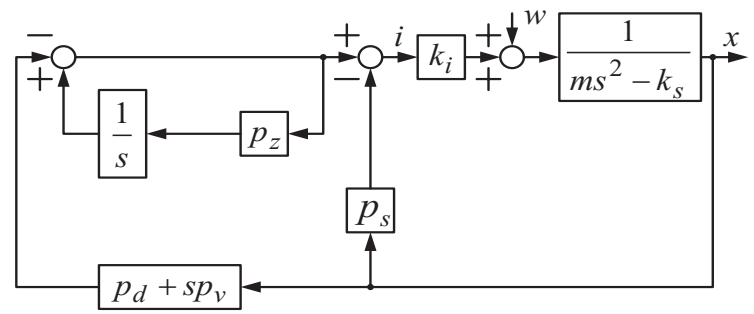

Fig. 3 Block diagram of the modified zero-power controller that can adjust stiffness

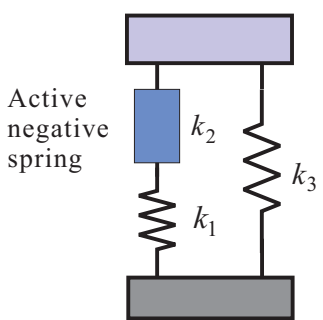

Fig. 4 Vibration isolation concept with negative stiffness

$$
\lim _{t \rightarrow \infty} x(t)=\lim _{s \rightarrow 0} s X(s)=-\frac{d_{0} p_{z}}{a_{0} p_{z}-b_{0} p_{s} p_{z}} F_{0}=-\frac{F_{0}}{k_{s}-k_{i} p_{s}}
$$

Therefore, the stiffness of the modified system becomes

$$
k=-k_{s}+k_{i} p_{s} .
$$

It indicates that the stiffness can be increased or decreased by changing the feedback gain $p_{s}$.

\section{Development of Vibration Isolation System Using Negative Stiffness}

The vibration isolation system is developed to generate infinite (high) stiffness for direct disturbing forces and to maintain low stiffness for floor vibration. Infinite stiffness can be realized by connecting a mechanical spring in series with a magnetic spring that has negative stiffness $^{(12),(13)}$. When two springs with spring constants of $k_{1}$ and $k_{2}$ are connected in series, the total stiffness $k_{c}$ is given by

$$
k_{c}=\frac{k_{1} k_{2}}{k_{1}+k_{2}} .
$$

The above basic system has been modified by introducing a secondary suspension to avoid some limitations for system design and supporting heavy payloads ${ }^{(2)}$. The concept is demonstrated in Fig. 4. A spring $k_{3}$ is added in parallel with the serial connection of positive and negative springs. The total stiffness $\widetilde{k}_{c}$ is given by

$$
\tilde{k}_{c}=\frac{k_{1} k_{2}}{k_{1}+k_{2}}+k_{3} .
$$

However, if one of the springs has negative stiffness that satisfies

$$
k_{1}=-k_{2} \text {, }
$$

the resultant stiffness becomes infinite for any finite value of $k_{3}$, that is

$$
\left|\tilde{k}_{c}\right|=\infty \text {. }
$$

This research applies this principle of generating infinite stiffness against direct disturbance to the system. On the other hand, if low stiffness of mechanical springs for system $\left(k_{1}, k_{3}\right)$ are used, it can maintain good ground vibration isolation performance as well. 


\section{Application of Feedforward Control}

To reduce the effect of transient response due to direct disturbance, a feedforward controller is added in combination with modified zero-power controller. Figure 5(a) shows the block diagram of the controllers with feedforward control. $H(s)$ indicates the feedforward compensator, which is defined as

$$
H(s)=\frac{q_{a} s}{s+q_{b}} .
$$

Therefore, the transfer function representation of displacement for the disturbance of the dynamics represented by Eq. (12) becomes

$$
\frac{X(s)}{W(s)}=\frac{\left(\left(1+k_{i} q_{a}\right) s^{2}+\left(q_{b}-p_{z}\right) s-q_{b} p_{z}\right) d_{0}}{\left[s^{3}+\left(b_{0} p_{v}-p_{z}\right) s^{2}+\left(b_{0} p_{d}+b_{0} p_{s}-a_{0}\right) s+a_{0} p_{z}-b_{0} p_{s} p_{z}\right]\left(s+q_{b}\right)} .
$$

To achieve the appropriate function of the compensator for disturbance suppression in the transient period for the system, values of the feedforward compensator becomes

$$
\begin{aligned}
q_{a} & =-\frac{1}{k_{i}}, \\
q_{b} & =p_{z} .
\end{aligned}
$$

It can be noted that, in many practical and industrial applications, direct disturbance on the table occurs due to the periodic load or repeated motions. The silicon wafer photolithography system, a principal equipment in the semiconductor manufacturing process, is an example of such application. It has a stage which is moved in steps and caused direct disturbance on the table. Suppression of the transient response to such disturbance is a major problem in the process. Therefore, the disturbance can be predicted well in advance because of the similar nature of disturbances. In such a case, when the external disturbance, $k_{d}$ is known, the parameter in Eq. (21) can be modified as

$$
q_{a}=-\frac{k_{d}}{k_{i}}
$$

Moreover, it can be mentioned that, the present vibration isolation system is fabricated by combining an upper suspension of negative stiffness and lower suspension of positive stiffness. The upper and lower suspensions are connected in series. Therefore, the current-force coefficient, $k_{i}$ in Eq. (23) is the summation of current-force coefficient of the upper actuator and that of the lower actuator.

It is worth noting that the PD controller and modified zero-power controller have limitations for excessive payload on the table. When the payload is very high, any one controller of the zero-compliance system might be unstable.

Therefore, it is a proposal for future work that a switching controller can be designed such that the PD controller and the zero-power controller will be switched to two separate I-PD controllers when excessive payloads act on the isolation table. Fig. 5(b) shows the proposed I-PD controller in combination with feedforward control. It can be mentioned that when $p_{z}=0$ in both Figs. 5(a) and (b), it becomes PD controller with feedforward control. 


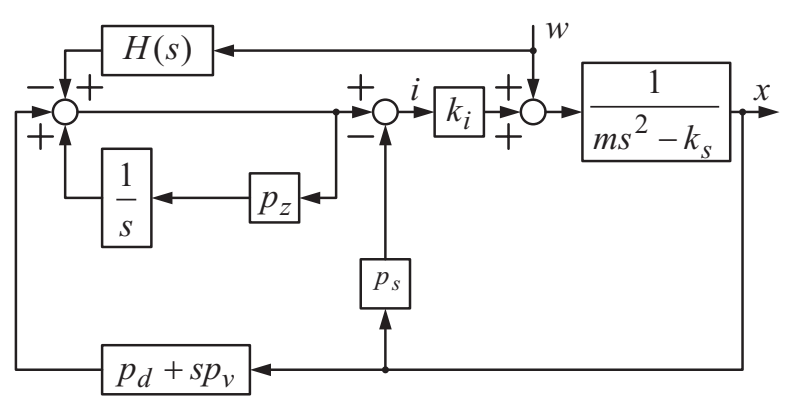

(a) Feedforward control with modified zero-power controller

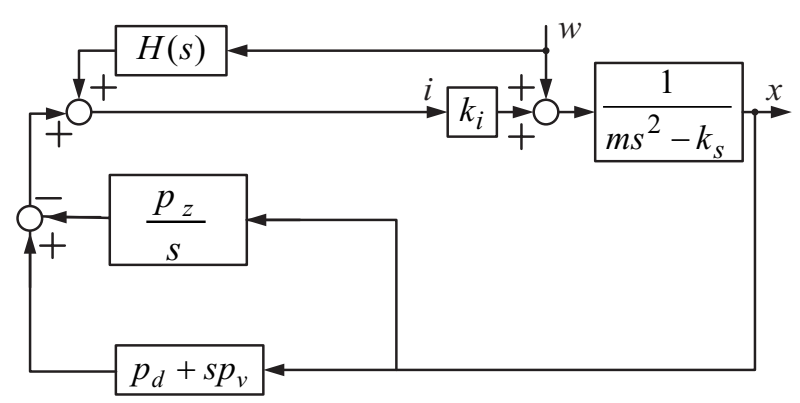

(b) Feedforward control with I-PD controller

Fig. 5 Block diagram of the feedforward controller with modified zero-power control and I-PD control

\section{Experimental Setup}

In order to verify the above analysis, a single-axis vibration isolation system was used. Figure 6 shows the experimental setup combining positive and negative stiffness suspension in series. It consisted of a circular base, a circular middle table and a circular isolation table. The height, diameter and weight of the system were $300 \mathrm{~mm}, 200 \mathrm{~mm}$ and $20 \mathrm{~kg}$, respectively. The relative displacement of the base to middle table was measured by an eddy-current displacement sensor, and positive stiffness was realized by a hybrid magnet consisted of an electromagnet (180-turns) that was fixed to the base, and four permanent magnets $(15 \mathrm{~mm} \times 2 \mathrm{~mm})$ attached to the middle table. The permanent magnets are made of two-pole cylindrical Neodymium-Iron-Boron $(\mathrm{NdFeB})$. The middle table was also supported by three coil springs. The hybrid magnet and the coil springs were used in tandem to generate positive stiffness suspension. Another displacement sensor was used to measure the relative displacement between the middle table and the isolation table. A different hybrid magnet, consisted of an electromagnet and six permanent magnets, was used to realize negative stiffness. The isolation table was also supported by three coil springs as weight support mechanism.

The motion of the isolation table and that of the middle table were restricted to move only in the vertical direction. It was done by using a vertical shaft which was fixed to the base and passed through the center of the isolation table and middle table. The friction between the shaft and the isolation table and middle table were reduced to a minimum possible level by using suitable lubricant and ball bearings. Two additional threaded shaft and several hexagonal nuts were employed as stopper and limiter for the movement of the isolation table and the middle table. Two leaf springs were used to confine the rotational motions of the isolation table and middle table. These leaf springs behaved as damper as well for the both tables. 


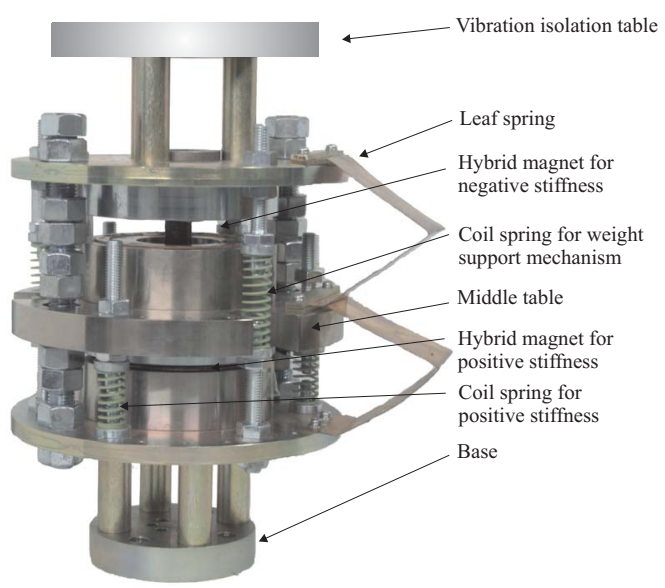

Fig. 6 Photograph of the vibration isolation system

\section{Experimental Results}

The experiments have been conducted to confirm the above mentioned analysis with the inclusion of feedforward control in the zero-compliance system. At first, the middle table was fixed with base, and the isolation table was levitated by PD control (Eq. (3) with or without Eq. (19) as shown in Fig. 2 when $p_{z}=0$ ). Similarly, the table was levitated subsequently by modified zero-power control (Eq. (11) with or without Eq. (19) as shown in Fig. 3) and I-PD control (Fig. 5(b) with or without Eq. (19)). Figure 7 shows the step response characteristics of the separate system with PD control, modified zero-power control and I-PD control, respectively. In this case, the disturbance was generated by an additional electromagnet placed above the table and the responses were captured by a digital oscilloscope. It is seen from the figures that peaks were appeared at the beginning and the end of step disturbances. This is occurred because an active feedback control may not achieve sufficient high-speed responses, due to the delays contained inherently in the control scheme. To diminish this transient response, feedforward signal of disturbance presented in Eq. (19) is introduced with the PD control, modified zero-power control and I-PD control separately. The results show that the peaks were duly suppressed when feedforward signal was added with the above controllers.

Next, the middle table was released, and then, the middle table and isolation table were suspended by PD control to achieve positive stiffness suspension. After that, isolation table was levitated by modified zero-power control to achieve negative stiffness. Zero-compliance of the isolation table (Eqs. (3) and (11) as shown in Fig. 4) was realized when stiffness of the upper suspension and lower suspension were equal, as described in Eq. (17). In this case, the negative stiffness was adjusted by modified zero-power controller. Figure 8 shows the step response of the isolation table using zero-compliance control. It is clear from the figure that the isolation table realized zero-compliance to step response. However, large peaks were emerged at the beginning and end of the steps. These peaks are the combined effect of PD control and modified zero-power control. The isolation table moved in the direction of load removal, and returned to the original position after certain period of time. When the feedforward controller was included in the zero-compliance control, the peaks were almost suppressed and response times were reduced to a minimum level. However, some minor vibrations were also noticeable in the figure, even after feedforward control. This is appeared mainly due to the disturbance signal of the electromagnetic actuator. The existing setup can control the motion in the vertical direction only. Therefore, the vibrations can be further reduced, if the isolation table is properly 


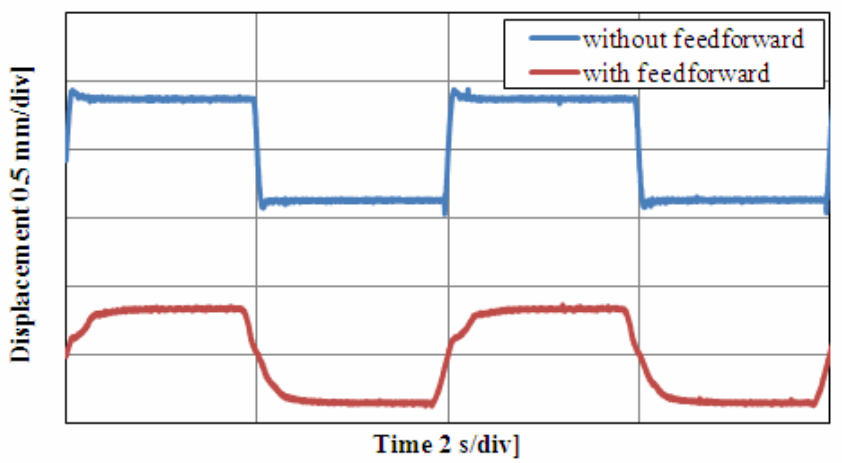

(a) PD control

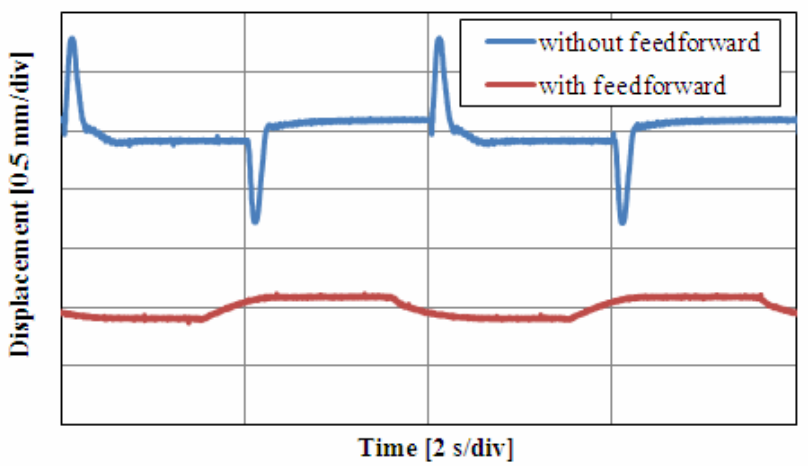

(b) Modified zero-power control

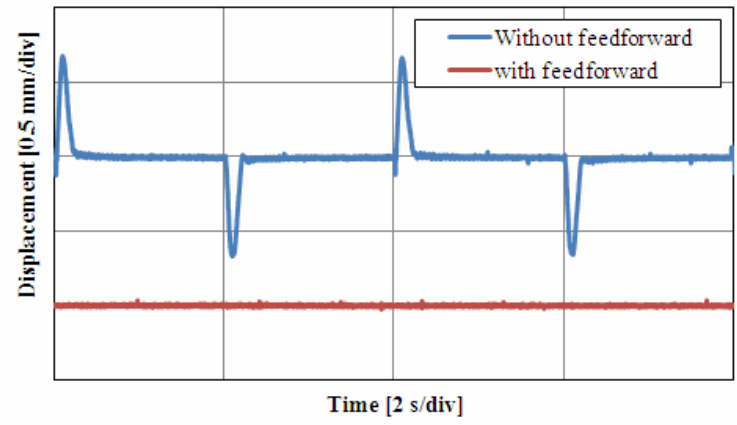

(c) I-PD control

Fig. 7 Step response of the table levitated with different controllers in combination with and without feedforward control

damped and the disturbance actuator is placed exactly on the center of the isolation table. Apart from this, nonlinearity of the zero-power control might be a problem in this case, because, the overshoots above and below the original position were not of same magnitude. Therefore, an improved controller with feedforward control, nonlinear compensation to zero-power control can solve such problem instead of using merely feedforward control.

Finally, the frequency response of the isolation table is measured with and without using feedforward control. The results are demonstrated in Fig. 9. It is obvious from the figure that the resonance peak and displacement of the isolation table was further reduced at the range below $30 \mathrm{~Hz}$ when the feedforward control included. 


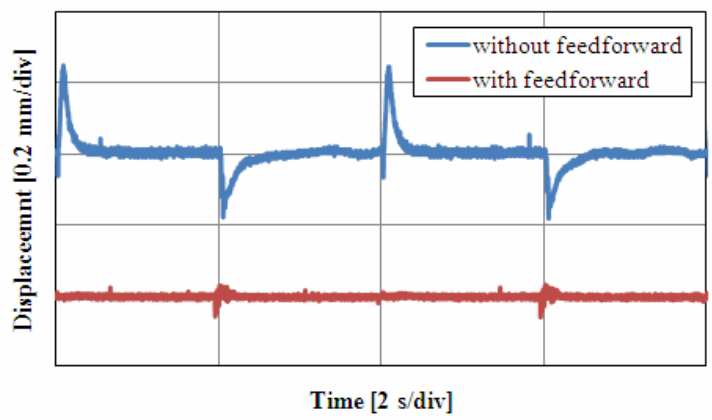

Fig. 8 Step response of the isolation table with zero-compliance control with and without feedforward control

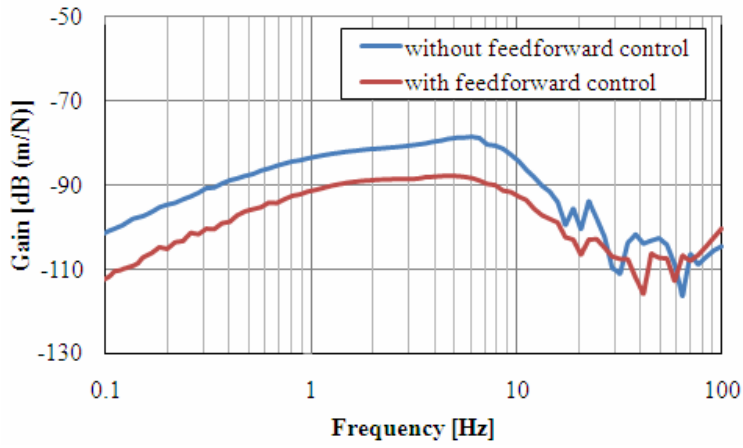

Fig. 9 Frequency response of the isolation table with zero-compliance control with and without feedforward control

\section{Conclusions}

A vibration isolation system has been developed using zero-power control in combination with feedforward control. The vibration isolation system was constructed by connecting a negative stiffness suspension by zero-power control in series with a positive stiffness suspension realized by PD control and coil springs. The zero-power control is modified by introducing a proportional displacement feedback to the zero-power control current. The modified zero-power controller yielded negative stiffness with the capability of adjusting negative stiffness. Feedforward controllers were added with both the modified zero-power control and PD control.

The results demonstrated that the proposed feedforward controller with the conventional controllers can suppress the peak brought up in response to direct disturbances in the vibration isolation system. It is expected that the resonance peak of the isolation table for ground vibration would be suppressed properly if such feedforward controller is added.

It can be noted that the payload supporting capacity of the zero-compliance system can further be improved if both controllers in the upper and lower suspensions are switched to I-PD control. The results also reveal that the feedforward controller in combination with I-PD control can compensate the overshoot appeared in response to step load. 


\section{Acknowledgment}

The research is funded by the Japan Society for the Promotion of Science (JSPS) as a Grant-in-Aid, and also from the Ministry of Education, Culture, Sports, Science Technology of Japan, as a Grant-in-Aid for Scientific Research (B).

\section{References}

(1) Rivin, E. I., Passive Vibration Isolation, (2003), ASME Press, New York.

(2) Harris, C. M., Shock and Vibration Handbook, (1996), McGraw Hill, Fourth Ed., New York.

(3) Kobayshi, I., Hirai, H. and Kumagai, M., Development of an Active Vibration Canceller for Photolithography Equipment, Proceedings of the $67^{\text {th }}$ Mechanical Dynamics Symposium, pp.195-198, (in Japanese).

(4) Watanabe, K., Hara, S., Kanemitsu, Y., Haga, T., Yano, K., and Mizuno, T., Combination of $\mathrm{H} \infty$ and PI Control for an Electromagnetically Levitated Vibration Isolation System, Proceedings of the 35th IEEE Conference on Decision and Control, Kobe, Japan (1996), pp. 1223-1228.

(5) Benassi, L., Elliot, S.J., and Gardonio, P., Active Vibration Isolation Using an Inertial Actuator with Local Force Feedback Control, Journal of Sound and Vibration, Vol.276, No. 3 (2004), pp.157-179.

(6) Daley, S., Hatonen, J. and Owens, D.H., Active Vibration Isolation in a "Smart Spring" Mount Using a Repetitive Control Approach, Control Engineering Practice, Vol.14 (2006), pp.991-997.

(7) Yoshioka, H. and Murai, N., Active Microvibration Control System by Pole Placement Method Using Genetic Algorithm, Proceedings of the SPIE Conference on Smart Structures and Integrated Systems, Newport Beach, Californea, USA (1999), pp.980-986.

(8) Yoshioka, H., Takahashi, Y., Katayama, K., Imazawa, T. and Murai, N., An Active Microvibration Isolation System for Hi-Tech Manufacturing Facilities, ASME Journal of Vibration and Acoustics, Vol.123 (2001), pp.269-275.

(9) Yasuda, M. and Ikeda, M., Double-Active Control of Microvibration Isolation Systems to Improve Performances (Application of Two-Degree-of-Freedom Control), Transactions of the Japan Society of Mechanical Engineers, Series C (in Japanese), Vol.59, No.562 (1993), pp.1694-1701.

(10) Yasuda, M., Osaka, T. and Ikeda, M., Feedforward Control of a Vibration Isolation System for Disturbance Suppression, Proceedings of the 35th Conference on Decision and Control, Japan (1996), pp.1223-1228.

(11) Miyazaki, T., Mizuno, K., Kawatani, R. and Hamada, H., Consideration about Feedback Feedforward Hybrid Control for Active Control of Micro-Vibration, Proceeding of the Second International Conference on Motion and Vibration Control, Yokohama, Japan (1994), pp.29-34.

(12) Mizuno, T., Proposal of a Vibration Isolation System Using Zero-Power Magnetic Suspension, Proceedings of the Asia-Pacific Vibration Conference, (2001), pp.423-427.

(13) Hoque, M. E., Takasaki, M., Ishino, Y. and Mizuno, T., Development of a Three-Axis Active Vibration Isolator Using Zero-Power Control, IEEE/ASME Transactions on Mechatronics, Vol.11, No.4 (2006), pp.462-470.

(14) Sabnis, A.V., Dendy, J. B. and Schmit, F. M., A Magnetically Suspended Large Momentum Wheel, Journal of Spacecraft and Rockets, Vol.12, No.7 (1975), pp. 420-427. 
(15) Morishita, M., Azukizawa, T., Kanda, S., Tamura, N. and Yokoyama, T., A New Maglev System for Magnetically Levitated Carrier System, IEEE Transactions on Vehicular Technology, Vol. 38, No. 4 (1989), pp. 230-236.

(16) Mizuno, T., Furushima, T., Ishino, Y. and Takasaki, M., General Forms of Controller Realizing Negative Stiffness, Proceedings of the SICE Annual Conference 2007, Kagawa University, Japan, (2007-09), pp. 2995-3000.

(17) Mizuno, T. and Takemori, Y., A Transfer-Function Approach to the Analysis and Design of Zero-Power Controllers for Magnetic Suspension Systems, Electrical Engineering in Japan, Vol. 141, No.2 (2002), pp. 933-940.

(18) Hoque, M. E., Takasaki, M., Ishino, Y., Suzuki, H. and Mizuno, T., An Active Microvibration isolator With Zero-Power Controlled Magnetic Suspension Technology, JSME Internatinal Journal, Series C, Vol.49, No.3 (2006), pp.719-726.

(19) Ishino, Y., Mizuno, T. and Takasaki, M., Stiffness Control of Magnetic Suspension by Local Feedback, Proceedings of the European Control Conference 2009, Budapest, Hungary, (2009-08), pp. 3881-3886.

(20) Mizuno, T., Takasaki, M., Kishita, D., and Hirakawa, K., Vibration Isolation System Combining Zero-Power Magnetic Suspension with Springs, Control Engineering Practice, vol.15, No.2 (2007), pp. 187-196. 